\title{
Risk Assessment for Transformer Loading
}

\author{
Weihui Fu, Student Member, IEEE, James D. McCalley, Senior Member, IEEE, and Vijay Vittal, Fellow, IEEE
}

\begin{abstract}
A risk-based probabilistic method is presented to assess transformer loading capability, taking into account the probabilistic nature of time-varying loads and ambient temperature. A sample calculation, with both component level analysis and system level analysis, is given. Quantitative reference risk levels are obtained based on reference loading levels given by IEEE/ANSI C57.115-1991. It shows that our quantitative risk assessment is useful in assessing transformer loading and in aiding related decision making. An additional benefit is that it enables inclusion of transformer loading in composite system risk analysis.
\end{abstract}

Index Terms-Loading cycle, Monte Carlo simulation, probability, risk assessment, transformer loading capability.

\section{INTRODUCTION}

$\mathbf{I}^{\mathrm{N}}$ $\mathrm{N}$ TODAY's competitive electric energy market environment, the incentive to heavily load power transformers is being driven by the need to achieve increased profits and the related reluctance to invest in new facilities. Hence, there is considerable interest in identifying decision-making criteria so that they can be fully, but safely utilized [1], [2]. We address this issue by describing a method for computing risk as a function of transformer loading. The computed risk can be used to identify individual transformer loading limits. It can also be used, together with risk calculation for transmission line overload, voltage collapse, voltage out-of-limit, and transient instability to obtain a composite risk as a function of operating conditions [3].

The condition that limits the transformer loading capabilities is the temperature of the winding and the insulation [4]. This condition is characterized by the winding hottest-spot temperature (HST). The winding HST in the top or in the center of the high or low voltage winding is the worst (highest) temperature for which the transformer insulation system is subjected. It is a function of ambient temperature, load shape, and transformer characteristics. Higher winding HST causes degradation in the strength of the winding insulation material. High temperatures decrease the mechanical strength and increase the brittleness of fibrous insulation, increasing the potential for transformer failure. Gas bubbles may also form which facilitate the dielectric breakdown characteristic of the transformer oil.

Many studies have been done to explore the effects of high loading under the motivation of loading transformer beyond the nameplate rating. Most of these studies focus on improving HST calculation models or developing methods for assessing the influence of transformer thermal delays on short term high loading

Manuscript received June 1, 1999; revised December 20, 2000. This work was supported by the Electric Power Research Institute, Contract W08604-01, and National Science Foundation, Grant ECS9502790.

The authors are with Iowa State University (e-mail: wfu@iastate.edu; jdm@iastate.edu; vittal@iastate.edu).

Publisher Item Identifier S 0885-8950(01)06054-0.

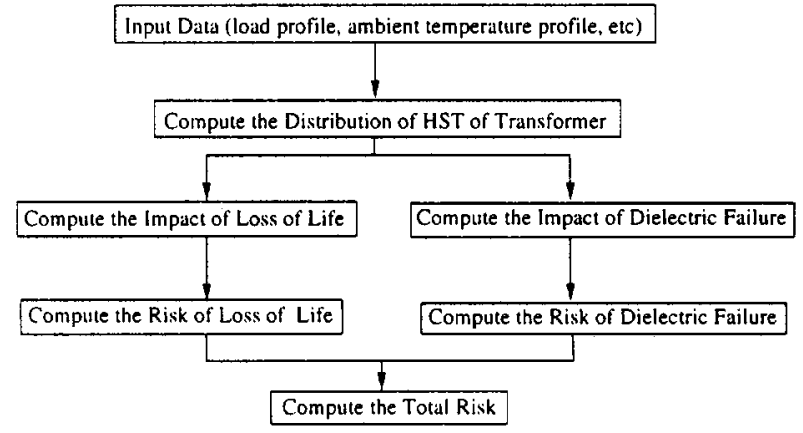

Fig. 1. The procedure for transformer risk calculation.

[5]-[9]. An advanced transformer diagnostic system including both software and hardware is described in [10], in which transformer loss of life and coil integrity could be predicted based on historical data. Reference [11] provides a transformer loadability study by simulating transformer thermal behavior considering the correlation between loading profiles of the transformer and ambient-temperature profiles. References [12] and [13] show some economic models to provide cost-benefit analysis of transformer overloading capability. References [14] and [15] give some practical guidelines for overloading the transformer based on both industrial standard and experience. All these studies are based on the assumption that the ambient temperature and loading profile are known; they do not take into account the probabilistic nature of time-varying ambient temperature and loads.

In Section II, we provide an overview of how we compute transformer risk, and Section III provides the corresponding analytical concepts. We identify reference risk levels corresponding to defined industry loading levels in Section IV. In Sections V and VI, we provide component level analysis and system level analysis to compute short-term risk and long-term cumulative risk. Section VII shows an example of composite system overload risk which includes the risk of transmission line and transformer overload, as a function of system load. Section VIII gives the conclusions.

\section{OVERVIEW}

In this section, we develop a systematic method to compute risk of transformer loading capability [16]. We treat the loads and ambient temperatures as uncertainties. A Monte Carlo technique is employed to calculate the probabilistic distribution of the winding HST. Based on the transformer HST, we calculate loss of life and failure probability of the transformer. The total risk for the transformer is obtained by summing the product of probability and consequence over all possible HST levels. We consider the consequence of a HST level in terms of loss of life and transformer dielectric failure. Fig. 1 shows this risk calculation procedure. 


\section{ANALYTICAL DEVElopmENT}

In this section, we develop a risk assessment model for transformer overloading.

\section{A. Hottest-Spot Temperature Model}

Transformer insulation deteriorates as a function of time and temperature [5]. Since the temperature distribution in most transformers is not uniform, the most common practice is to consider the aging effects produced by the winding hottest-spot temperature. We use the following method for calculating steady state and transient temperature, based on [17]-[19].

Given the transformer MVA loadings and the ambient temperature, the ultimate steady state top oil temperature rise $\theta_{u}$ over ambient temperature is computed as:

$$
\theta_{u}=\theta_{f l}\left(\frac{K^{2}+1}{R+1}\right)^{n} ; \quad K=\frac{S}{S_{\text {rated }}}
$$

where

$\theta_{f l}$ transformer top oil temperature rise over ambient temperature at "full" or rated load;

$K$ ratio of MVA loading to transformer nameplate rating (i.e., the ratio of load current to the rated load current if the voltage is assumed constant);

$R \quad$ ratio of loss at rated load to no-load loss;

$n$ exponential power of loss versus top oil temperature rise. $^{1}$

For transient temperature calculations, the top-oil temperature rise over ambient after $t$ hours is:

$$
\theta_{0}(t)=\theta_{u}\left(1-e^{-t / \tau_{0}}\right)+\theta_{i} e^{-t / \tau_{0}}
$$

where $\tau_{0}$ is oil thermal time constant for rated load, and $\theta_{i}$ is the initial top oil temperature rise over ambient. The HST rise above top oil temperature rise will be

$$
\theta_{g}(t)=\theta_{g(f l)} K^{2 m}
$$

where $\theta_{g(f l)}$ is hottest-spot conductor rise over top oil temperature at rated load, $m$ is the exponential power of winding loss versus winding gradient. ${ }^{2}$ Finally the HST $\theta_{h s t}(t)$ of the transformer after $t$ hours is

$$
\theta_{h s t}(t)=\theta_{0}(t)+\theta_{g}(t)+\theta_{a}(t)
$$

where $\theta_{a}(t)$ is the ambient temperature. If the initial top oil temperature $\theta_{i}$ is unknown, then it can be estimated based on the knowledge of 24-hour load cycle using the following iterative method.

- Step 1: Assume an initial HST.

- Step 2: Follow the preceding procedure to calculate the HST of each hour, including the HST of the 25th hour (first hour, next day).

- Step 3: Compare the assumed initial HST with the HST of the 25th hour. If they are close enough, stop; if not, use the 25th hour HST as the initial HST. Go to step 1.

Our experience indicates that usually, convergence is obtained in four to five iterations.

\footnotetext{
${ }^{1}$ Typical values are $n=0.8$ in the case of OA (self-cooled) operation; $n=$ 0.9 in the case of OA/FA (forced-air operation), OA/FA/FA; $n=1.0$ in the case of FOA (forced-oil-cooled), OA/FA/FOA, and OA/FOA/FOA operation [17].

${ }^{2}$ Typical values are $m=0.8$ in the case of OA, OA/FA, OA/FA/FA, and nondirected FOA, OA/FA/FOA, OA/FOA/FOA operation; $m=1.0$ for directed flow FOA operation [17].
}

\section{B. Uncertainties in Hottest-Spot Temperature Model}

When using the preceding model to calculate the transformer HST, there is typically some uncertainty regarding loading and ambient temperature. In the following, we provide probabilistic models to describe these uncertainties.

- Probabilistic Transformer Loading Profiles: Transformer daily load patterns in the future can be obtained by load forecasting, but load forecasting always has errors, and this error can be significant in today's deregulated environment. We assume that this uncertainty can be described by a normal distribution ${ }^{3}$ with the forecasted value as its mean [20].

- Distribution of Ambient Temperature: Similarly, for temperature uncertainty caused by weather forecasting error, we assume that it can also be described by a normal distribution with the forecasted value as its mean [21].

- Correlation between Loading Profiles and Ambient Temperature Profiles: It is observed that loading profiles are correlated with ambient temperature profiles [11]. For example, winter peak loads usually occur on the coldest days of the year; while summer peak loads occur on the hottest days of the year. So in winter the correlation between load and temperature should be negative; in summer it should be positive.

\section{Obtaining Probabilistic Distribution of HST}

In this section, we obtain the probabilistic distribution of transformer winding HST $\theta_{h s t}$. There are generally two kinds of approaches for obtaining distributions for parameters that are functions of random variables: analytical and Monte Carlo simulation. Monte Carlo simulations are typically more attractive than conventional analytic methods when dimensionality or model complexity make the analytical computation difficult. In this study, although either approach will work, we employ a Monte Carlo technique due to its mathematical simplicity and its ability to include more complex probabilistic models for the ambient temperature and transformer load. The calculation time for obtaining the HST probability distribution is not a serious concern as we can obtain a converged simulation result for one transformer in less than 1 minute on a Pentium $266 \mathrm{MHZ}$ PC for a 24-hour risk calculation.

Monte Carlo methods are based on the random sampling of scenarios, followed by the analysis of each sampled scenario [22], [23]. In our approach, we must obtain a distribution of ambient temperature and load over each hour. We assume this distribution is multivariate normal (MVN), and we generate it according to the following two steps:

- Step 1: Generate a univariate normal distribution $N(0,1)$. A simple scheme is the Box-Muller method [22]:

$X=\left(-2 \ln \left(V_{1}\right)^{1 / 2} \cos \left(2 \pi V_{2}\right) \quad Y=\left(-2 \ln \left(V_{1}\right)^{1 / 2} \sin \left(2 \pi V_{2}\right)\right.\right.$

where $X$ and $Y$ are a pair of independent random variables of $N(0,1)$, and $V_{1}$ and $V_{2}$ are a pair of independent random variables of uniform distribution $U(0,1)$.

\footnotetext{
${ }^{3}$ It is possible to choose any other distribution if it provides a better represen-
} tation of this uncertainty. 
- Step 2: Obtain the $2 T$ dimensional MVN distribution $Z$ by the following transformation

$$
Z=A Z_{0}+\mu
$$

where

$T \quad$ number of hours to be studied;

$Z_{0} \quad 2 T$ independent $N(0,1)$

$\mu \quad 2 T \times 1$ dimensional vector representing the means of the MVN distribution;

A $2 T \times 2 T$ matrix which satisfies $A A^{T}=\sum$;

$\sum$ covariance matrix characterizing the correlation between load and temperature over $T$ hours.

After sampling the ambient temperature and load in each hour, the HST in each sample is computed using the HST model described in Section III-A. Finally, we need to represent the simulation results of HST in each hour using the density function. The most commonly used nonparametric density estimator is based on the simple binomial distribution [27]. For a given $h$, we define $d_{i}$ as the number of observations falling in the temperature interval $\left(\theta_{h s t i}-h, \theta_{h s t i}+h\right)$. A natural estimator for probability density function which represents the hourly HST, is given by

$$
\hat{f}\left(\theta_{h s t i}\right)=\frac{d_{i}}{n}
$$

where $n$ is the total number of observations.

\section{Impact of Transformer Overloading}

In our approach, we consider two impacts due to the transformer overloading: loss of life and dielectric failure.

1) Loss of Life: The transformer insulation deteriorates as a function of time and temperature. The relation of insulation deterioration to changes in time and temperature is assumed to follow an adaptation of Arrhenius reaction rate theory as stated in the ANSI guides [17]-[19], which give the following expression for transformer insulation loss of life during time $t$ :

$$
D(\theta)=100 \times t \times 10^{-(A+B / \theta)} .
$$

Here, $D(\theta)$ is the percentage of total life lost, $\theta=\theta_{h s t}+273$ is the absolute temperature, $\theta_{h s t}$ is the hottest-spot temperature in ${ }^{\circ} \mathrm{C}$, and $A$ and $B$ are constants from the life expectancy curve [17].

If a transformer is operated within rated capacity, it could be reasonably expected to last in excess of 30 years if routine maintenance and testing are conducted. It is considered that the normal loss of life are from loadings that result in a daily loss of life equal to that of a continuously loaded winding hottest-spot temperature $\theta_{0}\left(110^{\circ} \mathrm{C}\right.$ for $65^{\circ} \mathrm{C}$ rise and $95^{\circ} \mathrm{C}$ for $55^{\circ} \mathrm{C}$ rise transformer, respectively)..$^{4}$ If the transformer hottest-spot temperature is higher than $\theta_{0}$, the aging rate of the transformer exceeds the normal value, which reduces the transformer working life and increases depreciation cost. So we consider the impact due to loss of life of thermal overloading is

$$
\operatorname{Im}_{1}(\theta)= \begin{cases}\frac{\Delta t}{t_{o}} \times C_{t} & \text { if } \theta>\theta_{o} \\ 0 & \text { otherwise }\end{cases}
$$

\footnotetext{
${ }^{4}$ Transformers with an average winding rise of not more than $65^{\circ} \mathrm{C}\left(55^{\circ} \mathrm{C}\right)$ and hottest-spot winding rise of not more than $80^{\circ} \mathrm{C}\left(65^{\circ} \mathrm{C}\right)$ are referred to as $65^{\circ} \mathrm{C}\left(55^{\circ} \mathrm{C}\right)$ rise transformers.
}

where

$$
\Delta t=D\left(\theta_{o}\right)-D(\theta)
$$

$t_{o} \quad$ expected percentage of transformer remaining life;

$C_{t} \quad$ cost of re-winding the transformer.

2) Dielectric Failure: For power transformers rated above 100 MVA, there are many other stress factors such as mechanical (short-circuit) stresses, bushing dielectric stress, and leakage flux density, all of which increase with transformer loading. If proper prevention measures are adopted, these factors are considered less severe than dielectric stress on insulation volume caused by the HST [17]. Therefore, we only consider the risk of dielectric failure caused by transformer overloading. ${ }^{5}$ It is believed that operating at insulated winding HST above $140{ }^{\circ} \mathrm{C}$ may cause gassing in the solid insulation and oil. Gassing produces a potential risk to the dielectric strength of the transformer. The test results in [24] indicate that there is a gradually increasing probability of dielectric failure whenever normal operating temperatures are exceeded, and the probability of failure is significantly increased at temperatures above $150^{\circ} \mathrm{C}$.

The probability of transformer failure during the next time period $\left[t_{0}, t_{1}\right]$ is

$$
\operatorname{Pr}\left(t_{0}<T \leq t_{1} \mid T>t_{0}, \theta\right)=\frac{\operatorname{Pr}\left(t_{0}<T \leq t_{1} \mid \theta\right)}{1-\operatorname{Pr}\left(T \leq t_{0} \mid \theta\right)} .
$$

We use the Hazard function to approximate the above probability. When $t_{0}$ and $t_{1}$ are close enough, eqt. (9) can be approximated by the following equation

$$
\operatorname{Pr}\left(t_{0}<T \leq t_{1} \mid T>t_{0}, \theta\right)=H\left(t_{0} \mid \theta\right) \times \Delta t
$$

where $\Delta t=t_{1}-t_{0}$ and $H\left(t_{0} \mid \theta\right)$ is the Hazard function

$$
H\left(t_{0} \mid \theta\right)=\frac{f\left(t_{0}\right)}{1-F\left(t_{0}\right)}
$$

where $f(\cdot)$ is the probability density function and $F(\cdot)$ is the cumulative distribution function of dielectric failure.

To compute eqt. (10), we need to know $f(\cdot)$ and $F(\cdot)$. Since Weibull Hazard plots have been successfully applied to analyze BPA transformer life data [25], we assume that the Weibull distribution can be used to describe the probability of transformer dielectric failure in its life cycle. Further, as we mentioned in Section III-D-1, the transformer insulation degradation is assumed to follow the Arrhenius relationship [17], which is a widely used model describing the effect that temperature has on the rate of a chemical reaction [27]. Therefore, we assume that the thermal related failure probability of a transformer can be described by the Arrhenius-Weibull models ${ }^{6}$ [26], [27], which is

$$
f(t)=\frac{1}{\sigma t} \phi(x) \quad F(t)=\Phi(x)
$$

where $\phi(x)=e^{x-e^{x}}, \Phi(x)=1-e^{-e^{x}}, x=((\log (t)-\mu) \sigma), \sigma$ is the scale parameter, $\mu=\mu_{\theta_{o}}-\log [A F(\theta)], \mu_{\theta_{o}}=11605 / \theta_{0}$, and $A F(\theta)$ is the Arrhenius acceleration factor which is

$$
A F(\theta)=\exp \left[E_{a}\left(\frac{11605}{\theta_{0}}-\frac{11605}{\theta}\right)\right] .
$$

${ }^{5}$ For cases when it is deemed necessary, risk of other factors may be included as well.

${ }^{6}$ This assumption could be verified experimentally or by analyzing existing data from industry. 
Here, $E_{a}$ is the activation energy in electron volts $(\mathrm{eV})$ of the insulation material, having range from 0.3 to 1.5 depending on the type of the insulation material [28].

A catastrophic failure of a transformer results in considerable costs. When such a failure occurs, the event is sudden, and the consequence can be substantial particularly if the system is stressed. The impact associated with transformer failure is the cost of replacing transformer capacity (including loss of load, loss in produced energy, process down-time and penalties). It can be quantified as

$$
\operatorname{Im}_{2}= \begin{cases}C_{\text {failure }} & \text { if failure } \\ 0 & \text { otherwise }\end{cases}
$$

where $C_{\text {failure }}$ is the cost of replacing transformer capacity due to the transformer failure.

\section{E. Risk Calculation}

Risk has been defined as the product of probability and impact [3]. The total impact of transformer thermal overload includes both the impact of loss of life and failure. Under a specified operating condition $X$ (typically characterized by transformer loading in terms of current) which is a function of time $t$, the risk over a period of $T$ is

$$
\operatorname{Risk}(\operatorname{Im} \mid X)=\operatorname{Risk}\left(\operatorname{Im}_{1} \mid X\right)+\operatorname{Risk}\left(\operatorname{Im}_{2} \mid X\right)
$$

where Risk $(\operatorname{Im} \mid X)$ is the risk corresponding to the operating condition $X$. The risk corresponding to loss of life is:

$$
\operatorname{Risk}\left(\operatorname{Im}_{1} \mid X\right)=\int_{0}^{T} \int_{\theta_{0}}^{\theta} \operatorname{Pr}(\theta \mid X) \times \operatorname{Im}_{1}(\theta) d \theta d t
$$

where $\operatorname{Pr}(\theta \mid X)$ is the probability density function of $\theta$ given $X$. The risk corresponding to transformer dielectric failure is:

$\operatorname{Risk}\left(\operatorname{Im}_{2} \mid X\right)=\int_{0}^{T} \int_{\theta_{0}}^{\theta} \operatorname{Pr}(\theta \mid X) \times H(t \mid \theta) \times \Delta t \times \operatorname{Im}_{2} d \theta d t$

\section{TRANSFORMER REFERENCE RISK LEVELS}

Power system load is typically cyclic in nature with both daily and annual cycles. For the daily cycle, it is usually assumed that transformers operate on a load cycle that repeats every 24 hours. The load cycle changes with seasons. It is usually appropriate to assume that the duration of the same load cycle extends over 90 days [8]. IEEE Standard C57.115-1991 [17] defines four types of loadings with progressively increasing degrees of risk (see Section IV-B). In this section, we use the approach developed in Section III to compute the risk associated with these types of loadings. We then use these risk levels as benchmarks on which to judge acceptability of transformer loading.

\section{A. Sample Data}

The sample transformer characteristics are shown in Table I, most of which are extracted from [17, Appendix C]. The transformer is rated FOA, $400 \mathrm{MVA}$, with $65^{\circ} \mathrm{C}$ average winding
TABLE I

TRANSFORMER CHARACTERISTICS

\begin{tabular}{c|c}
\hline Parameter & Value \\
\hline$\theta_{f l}$ & $36.0^{\circ} \mathrm{C}$ \\
$\theta_{g}(f l)$ & $28.6^{\circ} \mathrm{C}$ \\
$R$ & 4.87 \\
$\tau_{0}$ & $3.5 \mathrm{~h}$ \\
$n$ & 1.0 \\
$m$ & 0.8 \\
\hline
\end{tabular}

rise. Here, the parameters are defined as in Section III-A. We also make the following statistical assumptions:

- The deviation of loading profile is 10 percent of its mean.

- The deviation of ambient temperature is 30 percent of its mean.

- The correlation between load and ambient temperature is 0.01 .

In practice, one would obtain these values using historical data.

\section{B. Reference Loading Levels}

The following are four types of loading defined in IEEE Standard C57.115-1991 [17].

\section{- Normal Life Expectancy Loading}

Normal life expectancy loading (NLEL) is loading for which the winding HST and maximum top oil temperatures permitted in IEEE C57.12.00-1987 [32] are not exceeded, although the loading may exceed nameplate rating. This loading can be continued indefinitely; it is considered to be risk-free to remain in NLEL. To remain in NLEL, it is suggested that the winding HST be kept in the range of $110^{\circ} \mathrm{C}-120^{\circ} \mathrm{C}$.

- Planned Loading Beyond Nameplate Rating

Planned loading beyond nameplate rating (PLBNR) is loading for which the winding HST or top oil temperature exceeds the levels suggested for NLEL. It is accepted by the user as an anticipated, normal, reoccurring loading. This loading is allowed with all components in service, yet some risk is associated with. To remain in PLBNR, it is suggested that operation not exceed 4 hours per day when the winding HST is in the range of $120^{\circ} \mathrm{C}-130^{\circ} \mathrm{C}$.

- Long-Time Emergency Loading

Long-time emergency loading (LTEL) is loading for which the winding HST or top oil temperature exceeds those permitted for rated load operation. It is usually allowed only under conditions of prolonged outage of some system elements. To remain in LTEL, it is suggested one 24-hour period contains no more than six hours operation when the winding HST is in the range of $130{ }^{\circ} \mathrm{C}-140^{\circ} \mathrm{C}$, together with no more than four hours operation when the winding $\mathrm{HST}$ is in the range of $120^{\circ} \mathrm{C}-130^{\circ} \mathrm{C}$.

- $\underline{\text { Short-Time Emergency Loading }}$

Short-time emergency loading (STEL) is loading for which the winding HST or top oil temperature exceeds the limits given for PLBNR. It is an unusually severe condition typically acceptable only after the occurrence of one or more unlikely events that seriously disturb normal system loading. To remain in STEL, it is suggested that 
TABLE II

LIMITS FOR DIFFERENT TYPES OF LOADING ${ }^{7}$

\begin{tabular}{l|c|c|c}
\hline Types & $\begin{array}{c}\text { Winding } \\
\mathrm{HST} .{ }^{\circ} \mathrm{C}\end{array}$ & $\begin{array}{c}\text { Top oil } \\
\text { Temp. }{ }^{\circ} \mathrm{C}\end{array}$ & $\begin{array}{c}\text { Duration } \\
\text { (per day) }\end{array}$ \\
\hline NLEL & $110-120$ & 105 & $24 \mathrm{~h}$ \\
PLBNR & $120-130$ & 110 & $4 \mathrm{~h}$ \\
LTEL & $120-130$ & 110 & $4 \mathrm{~h}$ \\
& $130-140$ & & $6 \mathrm{~h}$ \\
STEL & $120-130$ & 110 & $4 \mathrm{~h}$ \\
& $130-180$ & & $1 \mathrm{~h}$ \\
& $130-140$ & & $6 \mathrm{~h}$ \\
\hline
\end{tabular}

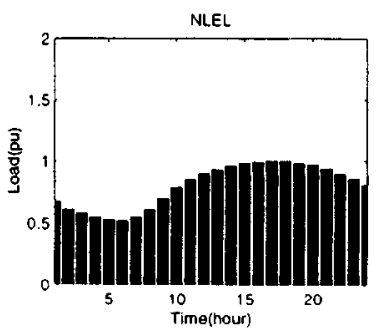

LTEL
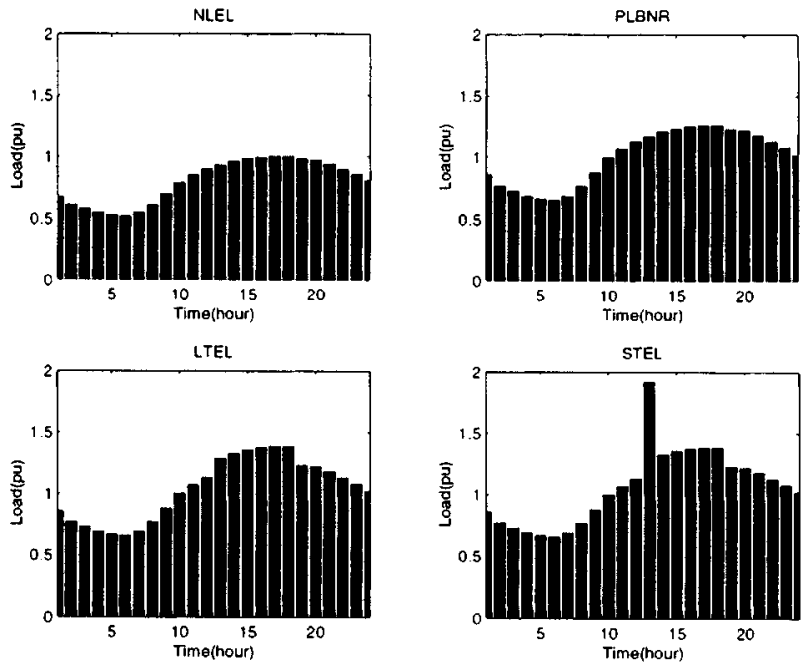

Fig. 2. Four types of loading, in p.u. of transformer rating.

one 24-hour period contains no more than one hour operation when the winding HST is in the range of $130^{\circ} \mathrm{C}-180^{\circ} \mathrm{C}$, together with no more than six hours operation when the winding HST is in the range of $130{ }^{\circ} \mathrm{C}-140{ }^{\circ} \mathrm{Cand}$ no more than four hours operation when the winding $\mathrm{HST}$ is in the range of $120^{\circ} \mathrm{C}-130^{\circ} \mathrm{C}$.

Table II provides the maximum temperature and duration limits per day for the above four types loading suggested in [17]. Fig. 2 shows an example of these four types of loading for a sample transformer [17].

\section{Reference Risk Levels}

Fig. 3 shows the risk of each hour in one day for four different types of loading 8 for the sample transformer. It can be seen that in each day the highest risk always appears around the time with heaviest load. It also shows, as expected, that the risk increases with increasing load.

For example, the total risk for NLEL is only 0.010740 p.u.; while for STEL it is 0.611894 p.u.

${ }^{7}$ Limits for other metallic HST, which refers to the hottest-spots temperatures of all grounded metallic structural parts whether in contact with solid insulation or not, are also given in [17]. These limits are given because it is believed that gas evolved at other metallic hot-spots does contribute significant risk, especially under the condition of LTEL and STEL [29]-[31]. We do not consider its effect in our risk calculation.

${ }^{8}$ We normalize the impact by choosing the cost of replacing transformer capacity $C_{\text {failure }}=100$ and the cost of re-winding the transformer as $C_{t}=$ 1.00 .
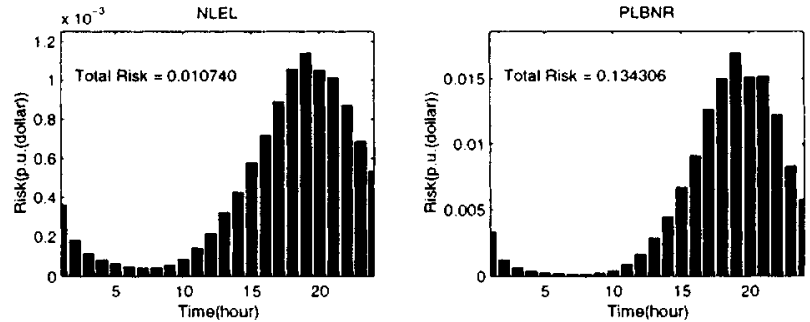

LTEL
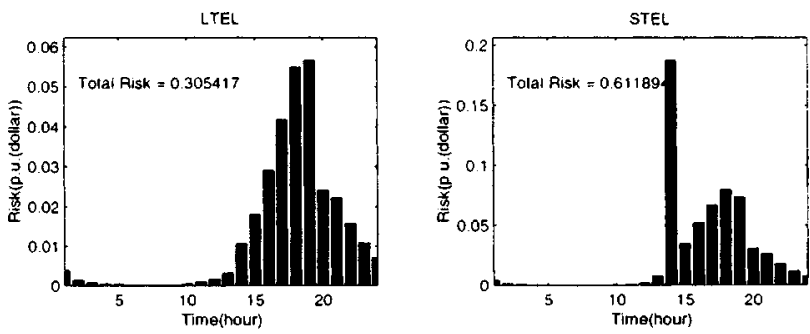

Fig. 3. Risk of different types of loading.

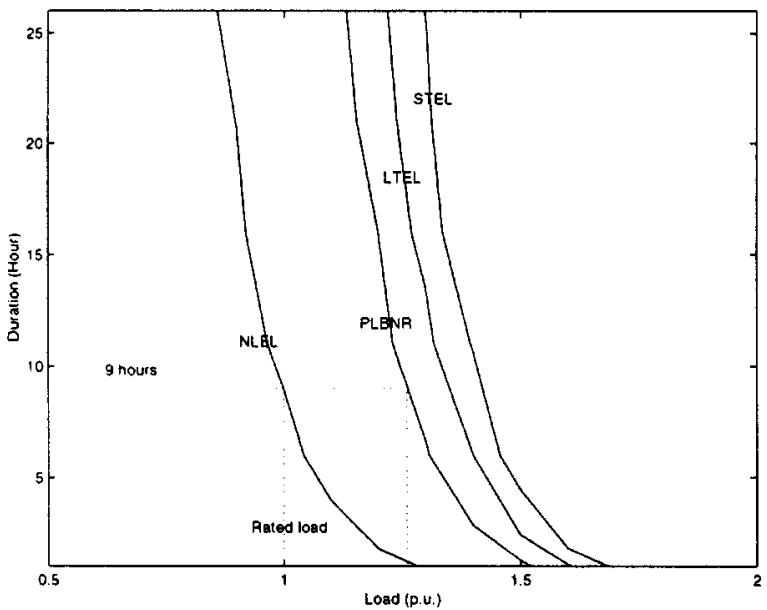

Fig. 4. Short-term loading capability based on equal risk criterion.

\section{COMPONENT LEVEl ANALYSIS}

In this section, we provide component level risk analysis to determine the transformer short-term loading capability and long-term loading capability.

\section{A. Short-Term Risk Assessment}

Fig. 4 provides iso-risk curves. The points on each curve have the same risk value. For example, the points on the curve NLEL have the same risk value as the loading profile NLEL, which is $0.010740 \times 10^{-3}$ p.u. Similar curves can be drawn for other risk values, if desired.

If one accepts that the risk of NLEL as the maximum risk value for the next 9 hours, then Fig. 4 indicates that the continuous loading of the transformer should not exceed its rated value. For one hour of loading, the same risk level would allow about $25 \%$ above nameplate loading. If one accepts the risk of PLBNR, then the transformer can be operated for 9 hours at $25 \%$ above nameplate loading.

\section{B. Cumulative Risk Assessment}

The method we developed can be used to compute cumulative transformer thermal risk over a specified time period. Some 


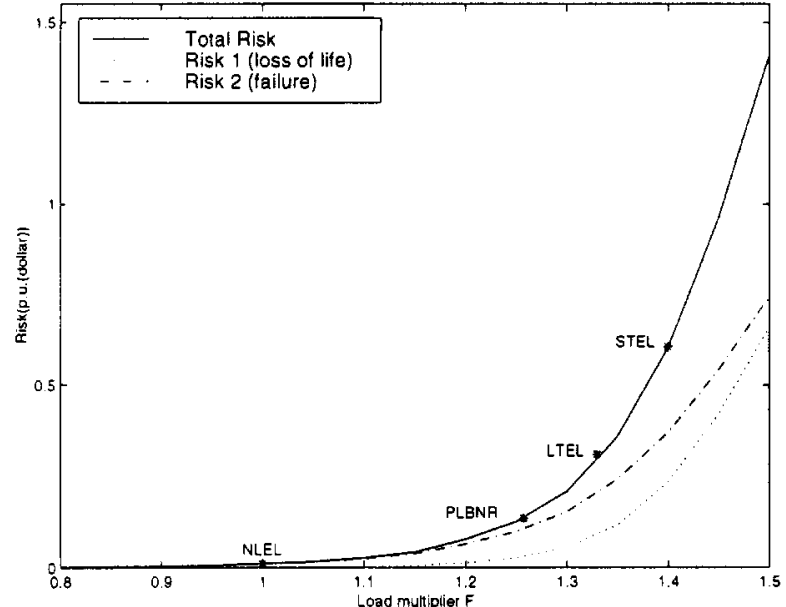

Fig. 5. Risk with different loading profile multipliers.

TABLE III

CORRESPONDING LOAD MULTIPLIERS FOR DIFFERENT TYPES OF LOADING

\begin{tabular}{c|cccc}
\hline & NLEL & PLBNR & LTEL & STEL \\
\hline $\mathrm{F}$ & 1.00 & 1.258 & 1.337 & 1.400 \\
\hline
\end{tabular}

transformers that serve to interconnect different portion of a transmission system may require studying over a time interval of a year or more to appropriately capture the risk variation, especially when the system is subject to uncertain flow patterns caused by the market. However, for transformers serving cyclic end-user demand, a time interval of 24 hours is sufficient to capture risk variation. We focus on this latter case to illustrate cumulative risk calculation for transformers.

We desire to enable identification of a load multiplier $F$, for a given repetitive 24-hour loading profile, corresponding to a maximum cumulative risk. Fig. 5 shows the risk of one day with different loading profile multipliers $F$ on NLEL. It can be used to identify how much additional risk is incurred as the demand grows. Conversely, it can be used to identify how much additional demand can be accommodated if one decide to accept an increased risk. For example, Table III gives the corresponding load multipliers, using NLEL as a base to maintain the same total risk as that indicated in Fig. 3 for each loading type.

\section{System LeVEL ANALYSIS}

There are five transformers altogether in the single area model of the IEEE RTS-96 system [33]. We assume all the transformers have the same characteristics and conditions as given in Section IV-A, and the same cost of transformer replacements for failures. The system load condition is normal winter weekdays. In order to accentuate the problem being studied, we modify the system by increasing the total system load to 110 percent, or 2172.56 MW. Fig. 6 shows the hourly loadings of five transformers of the IEEE RTS-96 system in normal winter weekdays.

Fig. 7 gives the quantitative risk of each hour for these five transformers. Since all the transformers are loaded far below their rated value (400 MVA), the risk values of these transformers are much lower than the reference risk levels developed in Section IV-C. But we still can observe that the trans-

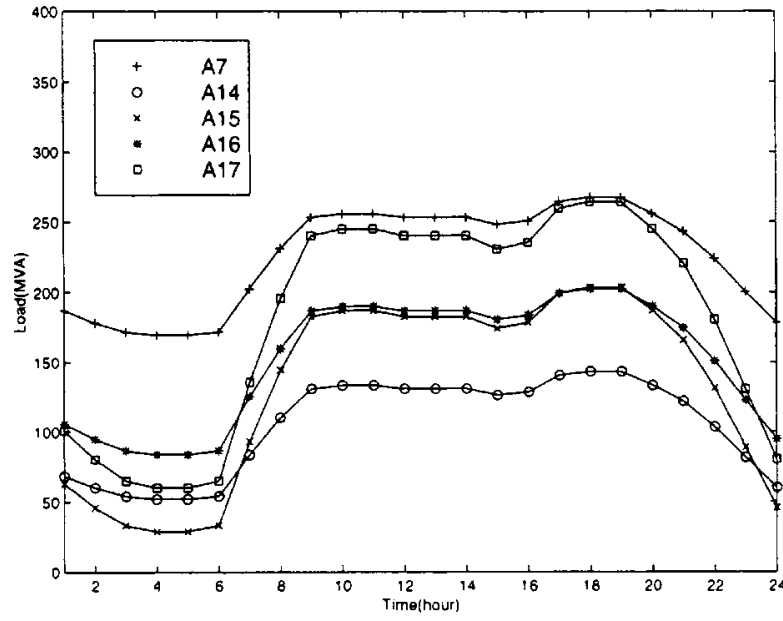

Fig. 6. Hourly load without outage.

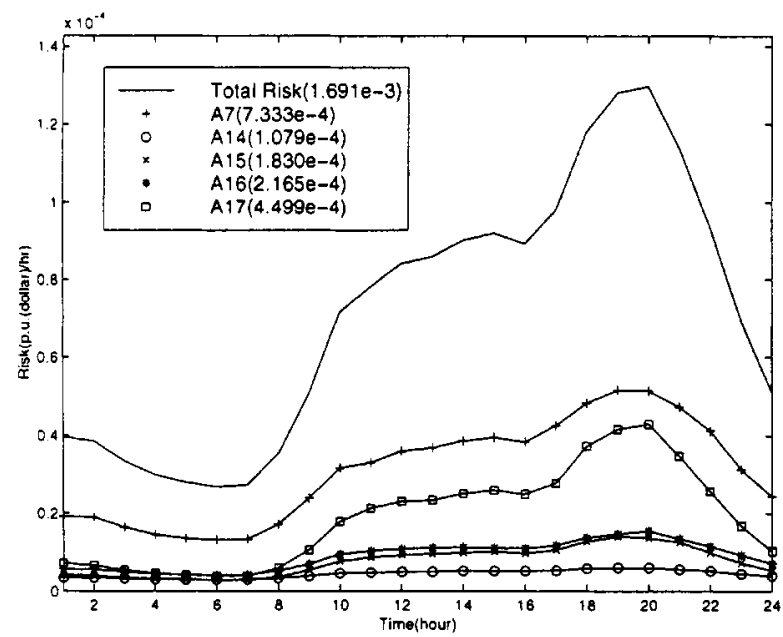

Fig. 7. Hourly risk without outage.

former A7 suffers the most risk of all five transformers since it has the highest loading level. In addition, we observe that small increases in loading can result in disproportionately large increases in risk, as illustrated by comparing the hour 20 loading peak with the hour 20 risk.

Figs. 8 and 9 give the results under a very serious operating condition when both transformer A16, in parallel with A17, and line 104-109 are outaged. Compared with the no outage case of Fig. 7, the total system transformer risk is increased about 7 times, and the risk related to transformer A17 is 1.190 e-2, which exceeds the reference risk for NLEL.

\section{COMPOSITE RISK ANALYSIS}

We have developed methods to compute the risk of transmission line overload [34], [35], voltage collapse [36], [37], voltage out-of-limit [36], [37], and transient instability [38]-[40] for defined operating conditions. Because our risk calculation for each problem type has the same unit, $\$ / \mathrm{hr}$, we can add them together to get a composite evaluation of security. Here, we illustrate by composing transmission line overload risk with transformer overload risk to obtain a total system overload risk. Fig. 10 shows the composite overload risk with system loading for the 


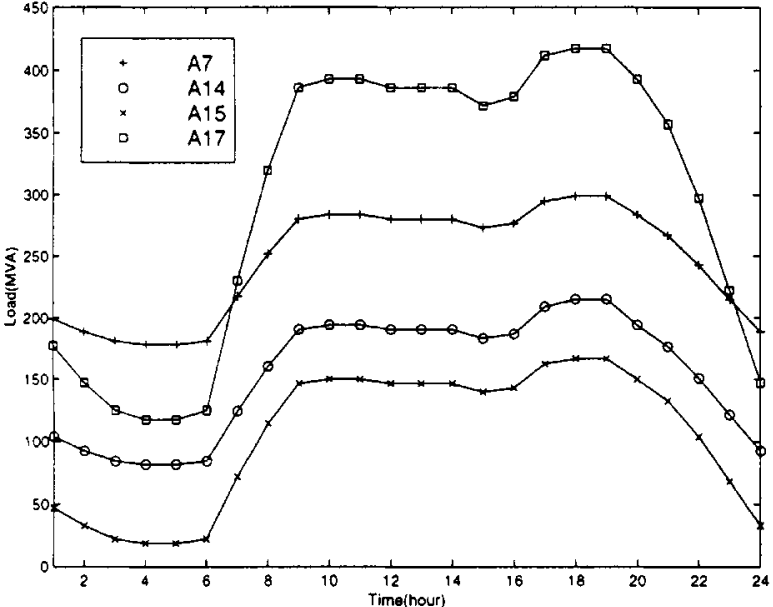

Fig. 8. Hourly load with outage A16 and line 104-109.

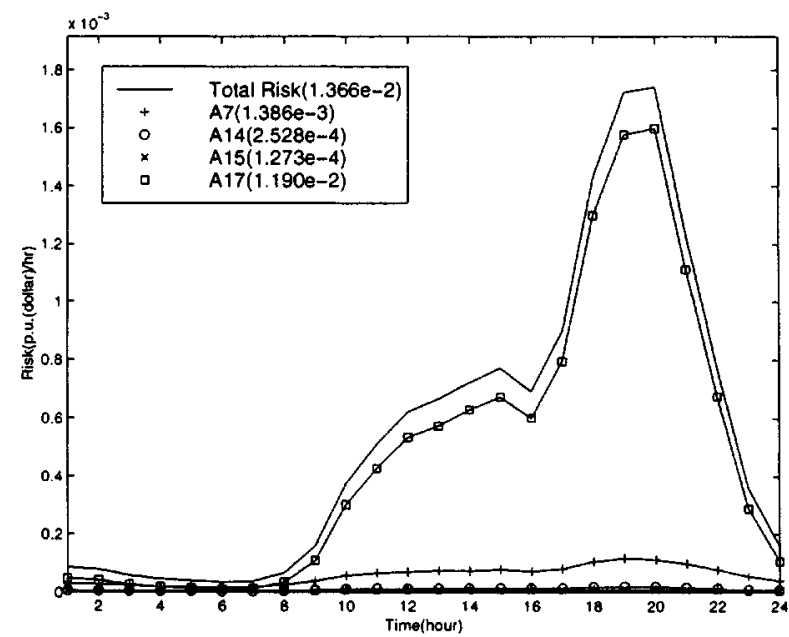

Fig. 9. Hourly risk with outage A16 and line 104-109.

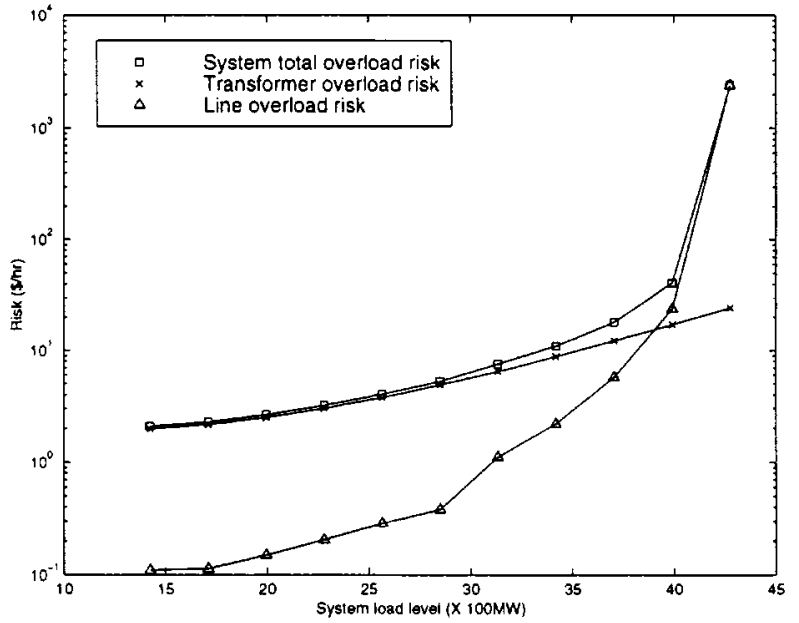

Fig. 10. System overload risk.

IEEE RTS-96. We assume that the system load and generation are proportionally increased. Calculation results show that loadings of the five transformers increase less than the loadings of lines 114-116 and 116-117, which are heavily stressed and dominate the transmission line risk. Thus, in Fig. 10, the total transmission line risk curve rises much more steeply than the total transformer risk curve.

Such an evaluation is useful to operators for monitoring overall system stress. In addition, it provides a much needed bridge between engineering reliability and market economics. We are currently developing a risk based optimal power flow to illustrate this bridge.

\section{CONCLUSION}

We provide a risk-based assessment method of transformer thermal loading capability. Compared with the traditional deterministic methods, this method has the following advantages:

- It determines a realistic estimate of transformer thermal loading capability by using probabilistic characterization of uncertainty rather than using conservative deterministic values.

- It provides a quantitative risk index that can be used to detect high risk situations.

- It can also be used, together with risk calculation for transmission lines overload, voltage collapse, voltage out-oflimit, and transient instability to obtain a composite risk as a function of operating conditions.

We believe this risk calculation method is helpful in making decisions related to balancing risk against the economic benefits that may result from a transformer loading level.

\section{REFERENCES}

[1] D. J. Allan, "Working transformer harder," in TechCon 98, 1998, pp. 39-45.

[2] C. Bengtsson, "Status and trends in transformer monitoring," IEEE Trans. Power Delivery, vol. 11, no. 3, pp. 1379-1384, July 1996.

[3] J. McCalley and V. Vittal et al., "Risk-based security assessment,", EPRI final report WO8604-01, Dec. 1998.

[4] R. R. Turcotte, "Causes of damage to power transformers," in TechCon 98, 1998, pp. 169-183.

[5] W. J. McNutt, "Risk assessment for loading transformer beyond nameplate rating," in Proceeding of American Power Conference, vol. 41, 1979, pp. 710-715

[6] W. E. Featheringill, "Power transformer loading," IEEE Trans. Industry Applications, vol. 19, no. 1, pp. 21-27, Jan./Feb. 1983.

[7] L. Pierce, "Predicting liquid filled transformer loading capability," IEEE Trans. Industry Applications, vol. 30, no. 1, pp. 171-178, Jan. 1994.

[8] T. T. Nguyen, "Constrained optimization procedure for evaluating cyclic loading of power transformers," IEE Proc.-Gener. Transm. Distrib., vol. 142, no. 3, pp. 240-246, May 1995.

[9] B. C. Lesieutre, W. H. Hagman, and J. L. Kirtley, Jr., "An improved transformer top oil temperature model for use in an on-line monitoring and diagnostic system," IEEE Trans. Power Delivery, vol. 12, no. 1, pp. 249-256, Jan. 1997.

[10] A. P. Meliopoulos, G. J. Cokkinides, and R. James, Jr., "An online transformer diagnostic system," in TechCon 98, 1998, pp. 23-35.

[11] C. C. Wong, "Substation power-transformer-loading analysis and computer simulations of loadability under realistic operating conditions," IEE Proc.-Gener. Transm. Distrib., vol. 141, no. 5, pp. 413-421, Sept. 1994.

[12] B. D. Lahoti and D. E. Flowers, "Evaluation of transformer loading above nameplate rating," IEEE Trans. Power Apparatus and Systems, vol. PAS-100, no. 4, pp. 1989-1998, Apr. 1981.

[13] Y. G. Bae, P. N. Adkins, and D. W. Bree, "Quantitative evaluation of the cost and benefits associated with transformer loading," IEEE Trans. Power Apparatus and Systems, vol. PAS-98, no. 1, Jan./Feb. 1979.

[14] P. K. Sen, "Understanding and assessment of transformer over-loading capability: An application guideline," in Proceedings of the American Power Conference, Chicago, 1996, pp. 968-973.

[15] J. O'Grady, “Overloading power transformers-A user's views," Power Engineering Journal, pp. 87-93, Mar. 1990. 
[16] W. Fu, J. McCalley, and V. Vittal, "Risk-based assessment of transformer thermal loading capability," in North American Power Symposium, Cleveland, OH, Oct. 19-20, 1998, pp. 118-123.

[17] IEEE Guide for Loading Mineral-Oil-Immersed Power Transformers Rated in Excess of 100MVA, IEEE/ANSI C57.115-1991.

[18] IEEE Guide for Loading Mineral-Oil-Immersed Power Transformers Up to and Including $100 \mathrm{MVA}$ with $55^{\circ}$ or $65^{\circ}$ average winding rise, IEEE/ANSI C57.92-1981 (Reaffirmed Jan. 1992).

[19] IEEE Guide for Loading Mineral-Oil-Immersed Overhead and Pad-Mounted Distribution Transformers Rated at 500kVA and Les. with $55^{\circ}$ or $65^{\circ}$ Average Winding Rise, IEEE/ANSI C57.91-1981 (Reaffirmed Jan. 1992).

[20] J. Mello and A. Melo et al., "Probabilistic evaluation of simulations transfer capability," in Proceedings of the Fifth International Conference of Probabilistic Methods Applied to Power Systems, Vancouver, Sept. 1997.

[21] S. Aboreshaid and R. Billinton, "Probabilistic evaluation of voltage stability,", 98 WM 981 PWRS.

[22] G. S. Fishman, Monte Carlo-Concepts, Algorithm, and Applications: Springer, 1996

[23] R. Billinton and W. Li, Reliability Assessment of Electric Power Systems Using Monte Carlo Methods: Plenum Press, 1994.

[24] W. J. McNutt and G. H. Kaufmann et al., "Short-time failure mode considerations associated with power transformer," IEEE Trans. Power Apparatus and Systems, vol. PAS-99, no. 3, pp. 1186-1197, May/June 1980.

[25] W. R. Vanderzanden, "BPA transformer life data analysis-Life cycle measures, uses and implications," in 10th Annual Conference on Reliability, Availability, Maintainability for the Electric Power Industry, Montréal, May 1983, pp. 8-14.

[26] W. J. McNutt, "Insulation thermal life considerations for transformer loading guides," IEEE Trans. Power Delivery, vol. 7, no. 1, pp. 392-401, Jan. 1992

[27] W. Q. Meeker and L. A. Escobar, Statistical Methods for Reliability Data: John Wiley \& Sons, Inc., 1998, to be published.

[28] W. Nelson, Accelerated Testing: Statistical Models, Test Plans, and Data Analysis: John Wiley \& Sons, 1990.

[29] IEEE Tutorial Course, "Power transformer considerations of current interest to the utilities,", 1984.

[30] IEEE Task Force Report, "Progress report on a guide for loading oil-immersed power transformers rated in excess of 100MVA," IEEE Trans. Power Apparatus and Systems, vol. PAS-100, no. 8, pp. 4020-4032, Aug. 1981.

[31] IEEE Power System Relaying Committee, "Adaptive transformer thermal overload protection,", Jan. 1999.

[32] IEEE Standard General Requirements for Liquid-Immersed Distribution, Power, and Regulating Transformers, IEEE/ANSI C57.12.00-1987.
[33] IEEE Task Force Report, "The IEEE reliability test system—1996,", 96 WM 326-9 PWRS

[34] H. Wan, J. McCalley, and V. Vittal, "Increasing thermal ratings by risk analysis," IEEE Trans. Power Syst., to be published.

[35] J. McCalley, V. Vittal, and N. Abi-Samra, "Overview of risk based security assessment," in Proceedings of the 1999 IEEE PES Summer Meeting, Edmonton, CA, July 18-22, 1999, to be published.

[36] H. Wan, J. D. McCalley, and V. Vittal, "Risk based voltage security assessment," IEEE Trans. Power Syst., to be published.

[37] J. McCalley, V. Vittal, H. Wan, Y. Dai, and N. Abi-Samra, "Risk based voltage security assessment," in Proceedings of the 1999 IEEE PES Summer Meeting, Edmonton, CA, July 18-22, 1999, to be published.

[38] J. McCalley, A. Fouad, V. Vittal, A. Irizarry-Rivera, B. Agrawal, and R. Farmer, "A risk based security index for determining operating limits in stability-limited electric power systems," IEEE Trans. Power Systems, vol. 12, pp. 1210-1219, Aug. 1997.

[39] V. Van Acker, J. D. McCalley, V. Vittal, and J. A. Pecas Lopes, "Risk based transient stability assessment," in Proceedings of the Budapest Powertech Conference, Budapest, Sept. 1999, to be published.

[40] J. McCalley, V. Vittal, V. VanAcker, and N. Abi-Samra, "Risk based transient stability assessment," in Proceedings of the 1999 IEEE PES Summer Meeting, Edmonton, CA, July 18-22, 1999, to be published.

Weihui Fu received the B.S. and M.S. degrees from Shanghai Jiaotong University, China, in 1991 and 1994, respectively. He is currently working toward the Ph.D. degree in electrical engineering at Iowa State University. He is a Student Member of the IEEE.

James D. McCalley is an Associate Professor of Electrical and Computer Engineering Department at Iowa State University, where he has been employed since 1992. He worked for Pacific Gas and Electric Company from 1986 to 1990. Dr. McCalley received the B.S. (1982), M.S. (1986), and Ph.D. (1992) degrees in electrical engineering from Georgia Tech. He is a registered professional engineer in California and a Senior Member of the IEEE.

Vijay Vittal is a Professor of the Electrical and Computer Engineering Department at Iowa State University. He received the B.E. degree (1977) in electrical engineering from Bangalore, India, the M.Tech. degree (1979) from the Indian Institute of Technology, Kanpur, India, and the Ph.D. degree (1982) from Iowa State University, Ames, IA. He is the recipient of the 1985 Presidential Young Investigator Award. He is a Fellow of the IEEE. 